\title{
Performance of Polarization-based Stereoscopy Screens
}

\author{
Xiaozhu Zhang, ${ }^{1}$ Kristian Hantke, ${ }^{1}$ Cornelius Fischer, ${ }^{2}$ and Matthias Schröter ${ }^{1,}$ 争 \\ ${ }^{1}$ Max Planck Institute for Dynamics and Self-Organization (MPIDS), 37077 Goettingen, Germany \\ ${ }^{2}$ Georg-August-Universität Göttingen, 3707ry Göttingen, Germany
}

(Dated: November 9, 2018)

\begin{abstract}
The screen is a key part of stereoscopic display systems using polarization to separate the different channels for each eye. The system crosstalk, characterizing the imperfection of the screen in terms of preserving the polarization of the incoming signal, and the scattering rate, characterizing the ability of the screen to deliver the incoming light to the viewers, determine the image quality of the system. Both values will depend on the viewing angle. In this work we measure the performance of three silver screens and three rear-projection screens. Additionally, we measure the surface texture of the screens using white-light interferometry. While part of our optical results can be explained by the surface roughness, more work is needed to understand the optical properties of the screens from a microscopic model.
\end{abstract}

\section{INTRODUCTION}

Displaying 3D content is not only an important issue in the entertainment industry, it is also of increasing importance in science where new numeric and experimental methods have created a wealth of three-dimensional datasets. Many stereoscopic display system are based on polarization filtering: the visual information for each eye is oppositely polarized, projected to and scattered or transmitted by the screen, and finally filtered by the viewer's glasses which consist of two polarizers admitting only the correctly polarized light to each eye [4, 5, 7]. There are two options for polarization filtering: linear and circular polarized light. While linear polarizers are simpler to manufacture, circular polarization has the advantage that head tilting will not impair the quality of the image

An ideal screen would completely preserve the polarization of the incoming light. However, in practice there is always some amount of "ghosting" resulting from the change of polarization at the screen. A measure for ghosting is the system crosstalk $C$. It is defined as the ratio between the intensity of light that leaks from the unintended channel to the intended one and the intensity of the intended channel 9 . According to measurements of Huang et al. 3], the maximal acceptable system crosstalk for a typical viewer to still experience a stereo sensation is 0.1 . (Lower values down to $10^{-4}$ can still be detected by careful visual inspection). While it is also known on a theoretical basis that the viewing angle will influence the amount of system crosstalk [8], to our knowledge no measurements of the angle-dependent system crosstalk of different screen types have been published up to now. Neither has the question been studied how the inclination angle (between the incoming light from the projector and the surface normal of the screen) influences the system crosstalk.

A second measure for the quality of a screen is the

\footnotetext{
* matthias.schroeter@ds.mpg.de
}

brightness of the image, which depends on the amount and angular distribution of the reflectance (for silver screens) or transparency (for rear-projection screens) of the screen. For silver screens this is typically quoted as the screen gain, the intensity measured at normal incidence normalized by the intensity of a Lambertian source 1. Here we measure the angle dependent scattering rate $S$ for both silver screens and rear-projection screens. $S$ is defined as the ratio of the intensity received by a viewer in a certain angle to the intensity of the incoming light, normalized by the solid angle.

In this paper we present measurements of the angular dependence of system crosstalk and scattering rate for three samples of silver screens (labeled SS1 to SS3) and three rear-projection screens (RP1 to RP3). Additionally, we determine the surface texture of the samples using white-light interferometry; this information provides some qualitative insight into our optical results.

\section{EXPERIMENTAL SETUP}

Figure 1 shows the experimental setup used for measuring the angular dependence of the system crosstalk and the scattering rate. Diode pumped solid state lasers (DPGL-2050 from Photop and Verdi V5 SF from Coherent) with a wavelength of $532 \mathrm{~nm}$ were used as light sources for the experiments. Passing a beam expander, the diameter of the laser beam was increased to $3.4 \mathrm{~mm}$ (FWHM), whereas the typical size of structural inhomogeneities on the screen surface is at most a few hundred micrometers as shown below. This ensured that the measured data for different spots on the screen are reproducible within $\pm 5 \%$.

The laser light was linearly polarized by passing a polarizer or circularly polarized by passing an additional Babinet-Soleil compensator (from B. Halle). The screen sample is irradiated by the laser at normal incidence and the scattered laser light of the silver and rear projection screens is detected by an detection unit in reflection (1b) and in transmission $(1 \mathrm{c})$, respectively. The detection unit consists of a power meter (PM100D with sensor S130C 


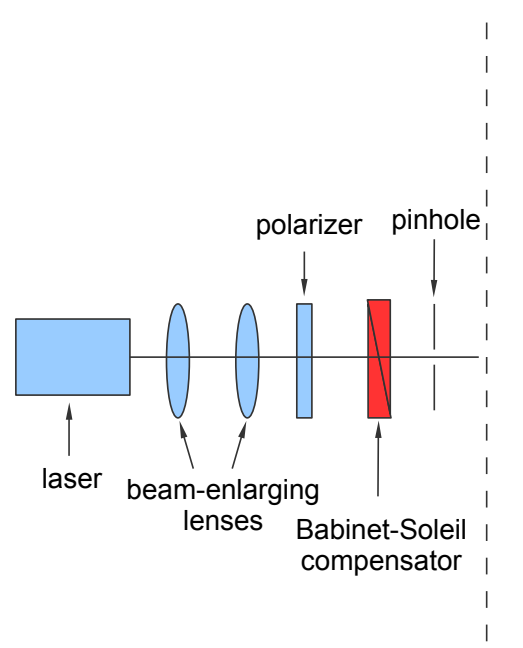

(a)

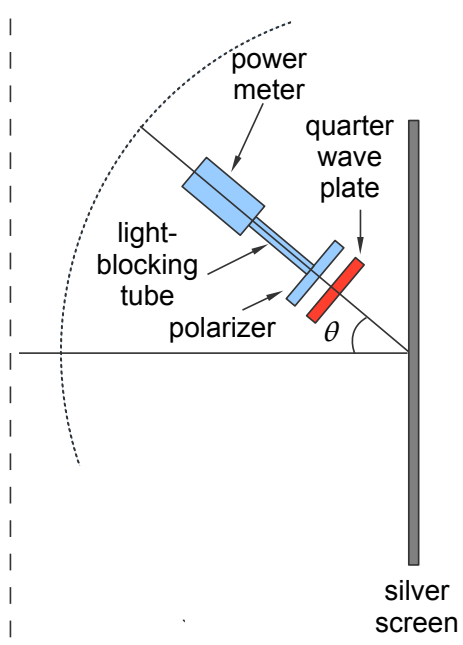

(b)

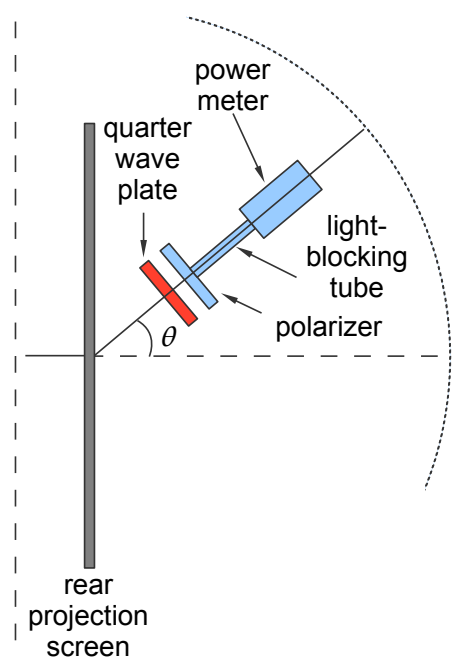

(c)

FIG. 1. Sketch of the experimental setup using circular polarized light: panels a) and b) are used for silver screens and a) and c) for rear-projection screens. The generation and the detection of linearly polarized light is achieved by removing the devices colored in red.

from Thorlabs), an analyzer and in case of the circular polarization an additional quarter-wave plate (both from B. Halle). A long and narrow tube was placed in front of the power meter, ensuring that only the photons are detected that scatter from the irradiated spot on the screen along the viewing axis of the sensor in a solid angle $\Omega$ of $2 \cdot 10^{-4} \mathrm{sr}$. The detection unit was placed on a rotatable rail with the rotational axis being fixed in such a way that the normal viewing axis of the sensor intercepts always with the illuminated area on the screen during rotation. The viewing angle $\theta$ can be varied from $-20^{\circ}$ to $80^{\circ}$. For silver screens the range of $\pm 6^{\circ}$ is inaccessible in order to not block the incoming beam.

In both cases of linear and circular polarization the incoming laser intensity $I_{\text {in }}$ was measured just in front of the sample. Furthermore, the intensity of the scattered light $I_{\text {out }}$ was measured for the intended channel with the polarization being the same direction as the incoming one ( $I_{\text {out }}^{s p}$, analyzer and polarizer parallel) and for the unintended channel with the polarization being the opposite direction $\left(I_{\text {out }}^{o p}\right.$, analyzer and polarizer perpendicular) for different viewing angles $\theta$. From this data one can compute the crosstalk $C(\theta)$ :

$$
C(\theta)=\frac{I_{o u t}^{o p}(\theta)}{I_{\text {out }}^{s p}(\theta)}
$$

and the scattering rate $S(\theta)$ :

$$
S(\theta)=\frac{I_{\text {out }}^{\text {sp }}(\theta)+I_{\text {out }}^{\text {op }}(\theta)}{I_{\text {in }} \Omega}
$$

The precision of the measurement for the crosstalk depends strongly on the purity of the initial laser polarization, whereas the scattering rate is not affected within our measurement precision. Analyzing the crosstalk without
TABLE I. Sample labels, brand names, manufacturer information on gain and transmission, and surface properties measured by white light interferometry: the root mean square roughness $R_{q}$ and the ratio between the surface area and the

\begin{tabular}{|c|c|c|c|c|c|}
\hline \multicolumn{2}{|c|}{ Sample|brand name } & gain & transmission & $R_{q}[\mu m]$ & $F$ \\
\hline RP1 & BS XRP3 & & 41.8 & 3.8 & 1.03 \\
\hline RP2 & WS XRP3 & & 88.8 & 3 & 1.13 \\
\hline RP3 & BS RP2 & & 41.2 & 4.2 & 1.2 \\
\hline $\mathrm{SS} 1$ & SH120 & 2.4 & & 5 & 2.3 \\
\hline SS2 & SF120 & 2.4 & & 8 & 2.6 \\
\hline SS3 & WA160 & 1.3 & & 22 & 2.2 \\
\hline
\end{tabular}
projected area $F$.

any screen sample (i.e. putting the laser directly in front of the analyzer system) we found the lower resolution limit in the linear case to be less than $3 \times 10^{-3}$. In the circular case the degree of polarization results in a lower resolution limit of $7.5 \times 10^{-3}$.

The screen samples were obtained from the company Screenlab (Elmshorn, Germany), their specifications and brand names are listed in table I

The surface topography of the screens was measured using a ZeMapper whitelight vertical scanning interferometer (Zemetrics, Tucson, USA): the focal plane of an interference pattern is vertically scanned through the sample topography, then a height map is calculated from the collected amplitude maps of the interference patterns. The vertical resolution of the instrument is better than $1 \mathrm{~nm}$; the maximum field of view applied in this study is $1.4 \mathrm{~mm}$. For more information on the instrument see [2. Prior to the measurement the rear projection screens where sputter coated with a $40 \mathrm{~nm}$ gold layer to increase surface reflectivity. 


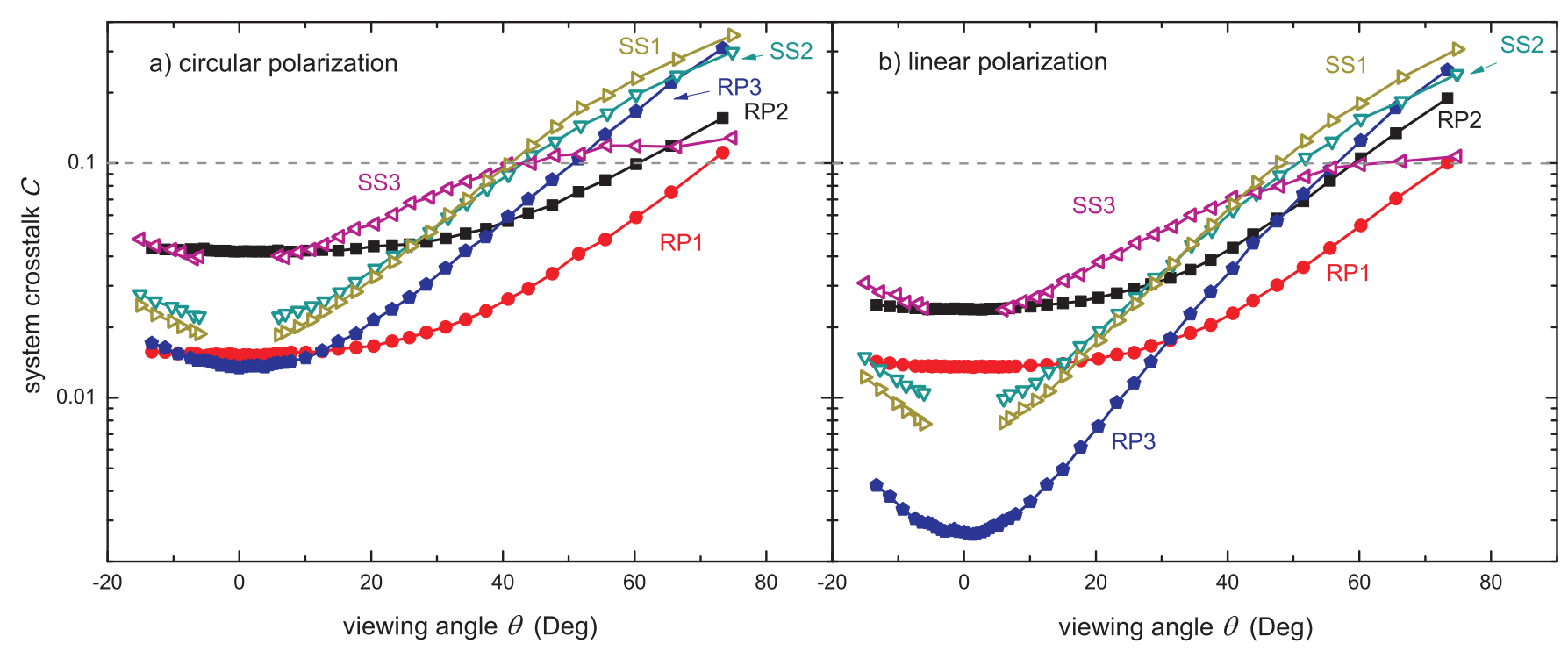

FIG. 2. Angular dependence of the crosstalk caused by the screen using a) circular polarized light and b) linear polarization. The grey dash line corresponds to the threshold for still acceptable stereo fusion according to Huang et al. 3 .

\section{RESULTS}

Figure 2 displays the system crosstalk of the six screen samples, both with circular and linear polarized light. Based on the criterion found by Huang et al. [3], all screens allow stereo vision for viewing angles $\theta$ smaller than $40^{\circ}$ (circular) or $48^{\circ}$ (linear). In practice this range will be smaller due to the additional crosstalk originating from the glasses and the inclination angle of the incoming light; the latter effect will be described below.

In general, the screens seem to fall into two categories; they are either optimized for a large range of acceptable crosstalk or a minimized crosstalk at small $\theta$. In both categories the silver screens are outperformed by the rear projection screens: RP3 has a smaller $C$ at small $\theta$ than SS1 while RP1 has a broader range of acceptable viewing angles than SS3.

Regarding the polarization mode, linear polarization has for each screen a clear advantage over circular. $C_{\text {circ }} / C_{\text {lin }}$ measured at $\theta=10^{\circ}$ varies between $1.1(\mathrm{RP} 1)$ and 4 (RP3) as shown in figure 3. Please observe, that our measurements of the crosstalk of RP3 at small angles might be limited by our experimental resolution.

Under real world conditions it is quite likely that the incoming light itself will have an inclination angle $\phi$ to the surface normal of the screen. To quantify the additional crosstalk created this way, we modified the experimental setup by adding a periscope in front of the polarizer. Figure 4 shows the crosstalk for sample SS1 in the case of linear polarization for inclination angles between $0^{\circ}$ and $15^{\circ}$. While there is a clear increase of crosstalk with $\phi$, the range of acceptable viewing angles $\theta$ is reduced by only $5^{\circ}$.

The scattering rates $S$ of the screen samples with circular polarization are shown in figure 5. Deviations of

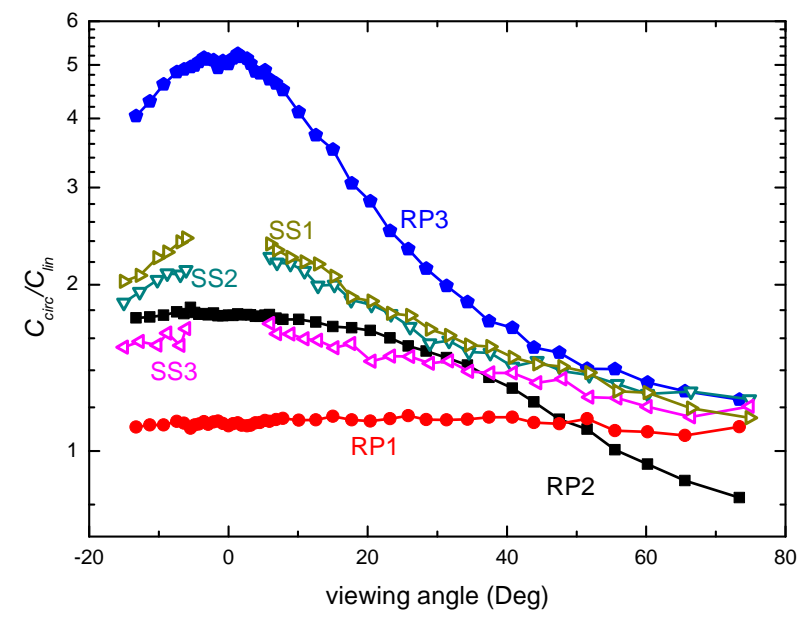

FIG. 3. Ratio of crosstalk measured with circular and linear polarization of the incoming light.

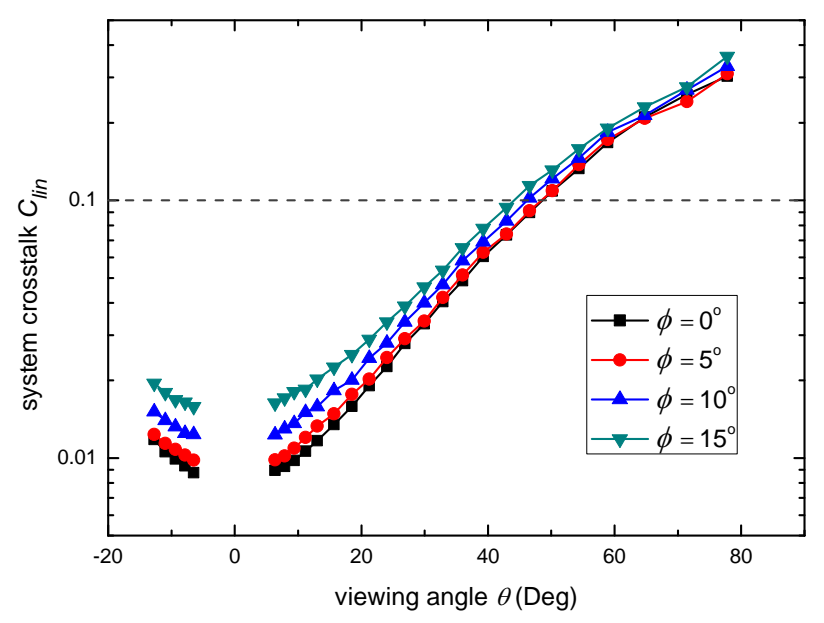

FIG. 4. Dependence of the crosstalk on the inclination angle $\phi$. Measured on screen SS1 using linear polarized light. 


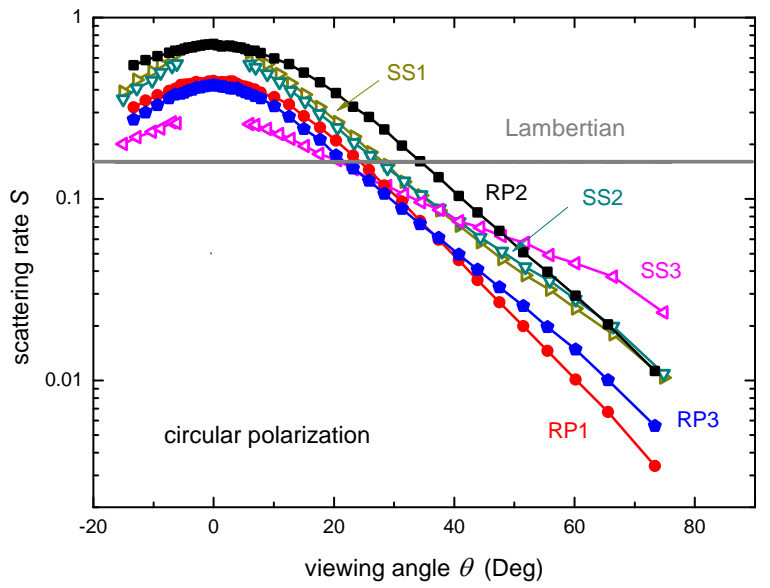

FIG. 5. Angular dependence of the scattering rate measured with circular polarization. Scattering rate values for linear polarization agree within 5.4 percent.

$S$ measured with linear or the circular polarization are within our errorbars. For high luminosities at small viewing angles SS1 and RP2 are the best choice, in terms of best homogeneity SS3 comes closest to a Lambertian source.

From a theoretical side the performance of a screen will depend both on its material and its surface texture [6].
While we do not have information on the electromagnetic properties of the screen material, the surface texture can be measured with white light interferometry. Perspective images of the surfaces of SS1, SS3, RP1, RP3 are shown in figure 6. The RMS (root mean square) roughness $R_{q}$ and the ratio between the surface area and the projected area $F$ of all six screen samples is listed in table $\mathrm{I}$.

A comparison of the angular dependence of $S$ with these values hints at $R_{q}$ as a predictor for the deviation from a Lambertian source. This is particularly shown by SS3 which has by far the highest value of $R_{q}$ and the smallest $\theta$ dependence of $S$. Regarding the system crosstalk a similar correlation between $R_{q}$ and the slope of $C$ at large angles might exist. On the other side we do not find a clear correlation between the optical properties and $F$.
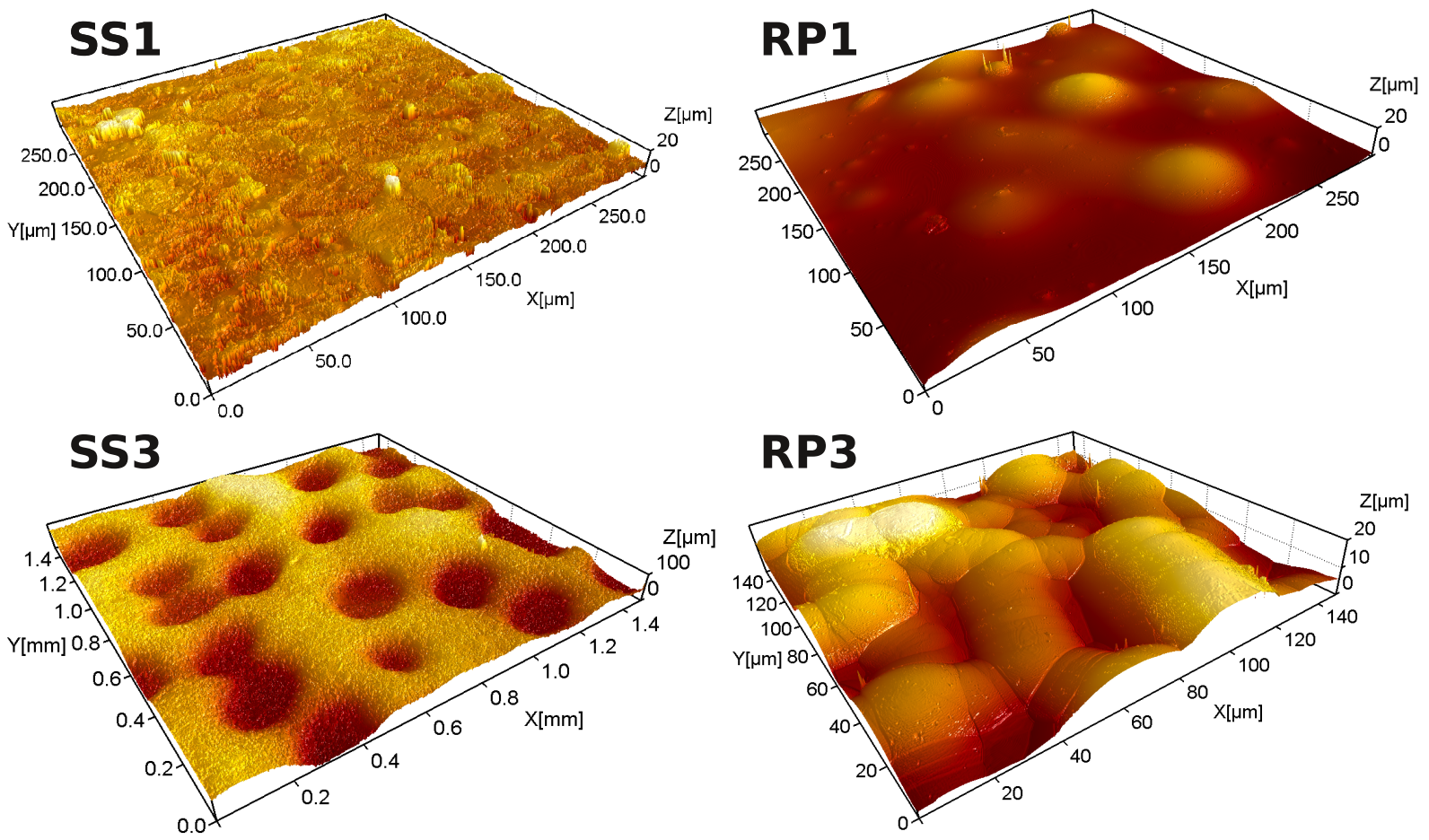

FIG. 6. Perspective images of the surface texture of the screen samples. Please note the different horizontal and vertical scales for sample SS3. Images contain between $0.7 \%$ (RP1) and 30\% (SS3) interpolated pixels. 


\section{CONCLUSION}

All screens allow effective stereo projection for viewing angles up to $40^{\circ}$. At larger angles the crosstalk of rear projection screens is considerably smaller than that of silver screens. Also for each screen the crosstalk was larger with circular polarization than with linear. However, when planing a display system additional factors have to be taken into account like the available space be- hind the screen or the sensitivity of the system against the viewers tilting their heads. Consequentially, no optimal solution for all possible scenarios exist. While the roughness of the screens influences their large viewing angle behavior, clearly more research is needed for a quantitative understanding.

Acknowledgements: We would like to thank Günter Daszinnies from the company Screenlabs for providing the test samples.
[1] Brennesholtz, M.S., Stupp, E.H.: Projection Displays. Wiley (2008)

[2] Darbha, G., Schäfer, T., Heberling, F., Lüttge, A., Fischer, C.: Retention of latex colloids on calcite as a function of surface roughness and topography. Langmuir 26, 4743-4752 (2010)

[3] Huang, K.C., Yuan, J.C., Tsai, C.H., Hsueh, W.J., Wang, N.Y.: A study of how crosstalk affects stereopsis in stereoscopic displays. In: Proceedings of SPIE-IS\&T, vol. 5006, pp. 247-253 (2003)

[4] Iizuka, K.: Welcome to the wonderful world of 3D: introduction, principles and history. Optics and Photonics News 17, 42-51 (2006)
[5] Janssen, J.K.: 3D 2.0, Neuer Anlauf für Stereoskopie im Kino. c't 16, 72-75 (2008)

[6] Jin, L., Kasahara, M., Gelloz, B., Takizawa, K.: Polarization properties of scattered light from macrorough surfaces. Optics Letters 35, 595-597 (2010)

[7] Kim, S.C., Kim, E.S.: Performance analysis of stereoscopic three-dimensional projection display systems. 3D Research 1, 1-16 (2010)

[8] Richards, M., Schnuelle, D.: The effective gain of a projection screen in an auditorium. SMPTE Motion Imaging Journal 119, $62-67$ (2010)

[9] Woods, A.J.: How are crosstalk and ghosting defined in the stereoscopic literature? In: Proceedings of SPIEIS\&T, vol. 7863, p. 78630 Z (2011) 